\title{
An analysis of the impact of reference groups on collectivist families' meal social interaction behaviour in Sierra Leone.
}

\author{
Sheku Kakay* \\ Department of Management, Leadership and Organisation, Hertfordshire Business School, University of Hertfordshire, \\ United Kingdom
}

\begin{abstract}
Background: Reference groups predominantly shapes the meal social interaction behaviour of most Sierra Leonean families as it is designed to orient their thinking about the symbolic role of hierarchy and authority, which help define the character of children as they grow-up and teaches them responsibility. The re-shaping and streamlining of the family members' character help foster a better relationship between them and promotes closer ties even with external relations, neighbours and others within the community. In addition, it significantly helps the family to plan its food purchase and consumption habits, which prevents them from experiencing food scarcity. However, reference groups impinge on the effective use of resources and food distribution in the family, which limits their ability to access variety, quality and adequate food. The differences in cultural backgrounds between family members and their extended relations impacts on the family negatively in terms of thieving, divulging of family secrets, backbiting and most significantly breeding jealousy and nepotism. This paper, therefore, critically evaluates the impact of reference groups on collectivist families' meal social interaction behaviour in the Sierra Leonean context.
\end{abstract}

Methods: The researcher used one-to-one semi-structured qualitative interviews to investigate families' views and experiences of their mealtimes' behaviours. In this research, due to the fact that the selected samples of families were unknown, the researcher used snowballing; convenience; and experiential sampling in recruiting respondents, including males and females from different cultural, ethnic, religious and professional backgrounds, across the different regions of Sierra Leone. The interviews were guided by a topic, and this procedure was followed until no new themes emerged. The interviews were recorded using an audio recorder, which were transcribed verbatim and analyzed using a thematic approach

Results: Twenty families (comprising 20 husbands and 20 wives) with a sample size of 40 participants were interviewed in this study. The paper highlights the influence of reference groups on the behaviour of Christian and Muslim families (husband and wife) at mealtimes and draw attention to its significance as influencer of collectivism, particularly in relation to its impact on the social interaction between similar and dissimilar gender groups. The author critically analyzed the influence of reference groups on families' meal social interaction behaviour and presents a comparative summary of how gender affects the meal behaviours of different gender and religious groups.

Conclusion: This study suggests that reference groups play a key role in influencing families' behaviour at mealtimes, which espouse positive and negative effects on their meal social interaction behaviours. To capture its symbolism among the different social groups, the author critically reviewed and presented a comparative analysis of the similarities and differences in behaviour between and across gender and religious groups.

Keywords: Collectivism/Individualism, Reference groups, In-group/Out-group, Social interaction, Consumer behaviour

\section{Background}

Reference groups, in the Sierra Leonean context, have predominantly influenced individuals' collectivist behaviours over the years and will continue to do so in the future, though discrepancy still exists among various theorists about their degree of influence $[1,2]$. Lawan et al. Ayoko et al. pointed to the tendency of focusing on group preferences and group harmony in collectivist cultures, which leads to the opportunity of suppressing internal (personal) attributes in certain settings
$[3,4]$. Abraham et al. and Bolten argued that, kinship networks are extremely important in the everyday matters of Sierra Leonean families, and that one is obliged to assist one's family members throughout life [5,6]. Accordingly, most people in Sierra Leone abide by the collectivist cultures because of the limited opportunity that exists in terms of jobs and other social amenities and therefore drive the collectivist tendency upwards to enhance the survival of close families, in-groups and/or kinship network. This collectivistic tendency is evident 
in almost all ethnic groups within Sierra Leone; except for a few people who are well educated and have attained certain social status in society employ the British or American individualistic orientations [7].

In Sierra Leone, whose culture is predominantly collectivist; the idea of accommodating extended family values is not uncommon-with one large joint family unit living together under the same roof with a single breadwinner. A number of theorists re-affirmed this argument and that the basic household structure of Sierra Leone is an extended family, organized for the majority of people around the farm and its rice production $[8,9]$. Chinunda reiterated that many households are polygamous, where a husband may have more than one wife; the first or "senior" wife usually has some authority over "junior" wives, such as in training and organizing them into a functional unit [10]. However, Manyama also proclaimed that monogamy is also common, especially among urban and Christian families [11]. Akin to this view, Sanchez-Sosa et al, Amato and Epstein suggested that the extended family provides a very different type of environment for interaction among members as there are multiple sources of influences based on interaction and observation of others, and the family members are considered as of greater importance than outsiders [12-14]. This evidence is glaring in the Sierra Leonean culture, where most family members show a high degree of commitment and loyalty for people of the same descendants/in-groups. However, as the generational link expands beyond two generations, the level of commitment, loyalty and unity declines, which can be largely attributed to acculturation [15-17].

The degree of influence of reference groups, particularly extended families on families' meal social interaction behaviour in the African context is yet to be firmly established, as there is a lack of empirical data/evidence to substantiate the claim. A number of theorists, for example, Attah et al., Daya et al. and Horner et al, affirmed that the concept and impact of extended families or reference groups on the food consumption behaviour of people in the African continent is yet to be fully studied [18-20]. They emphasized that, most literature on consumer behaviour focused either on the United States, United Kingdom and other European countries or on China, Japan and other Asian countries. It is against the backdrop of these arguments that the researcher evaluated the impact of reference groups on families' meal social interaction behaviour in the Sierra Leonean collectivist context.

\section{Methods}

The researcher conducted one-to-one semi-structured face-toface qualitative interviews with families about the reference group factors that influenced their meal social interaction behaviour. This allowed families from different ethnic, religious and cultural backgrounds, based on their perspective and own words elucidate their views of the reference group attributes that influences their meal social interaction behaviours. The researcher during the semi-structured interviews introduced a theme and allowed the conversation to develop according to cues taken from what respondents said about their families.

\section{Participants and recruitment}

The researcher used snowballing; convenience; and experiential sampling to recruit families from different ethnic and religious (Muslim and Christian) backgrounds from across the four regions of Sierra Leone, including the northern, southern and eastern provinces as well as the western area. The researcher primarily focused on urban areas, particularly the provincial headquarter towns with about $20 \%$ of the families selected in the North (Makeni), 20\% in the South (Bo), 20\% in the East (Kenema), and $40 \%$ in the Western area (Freetown). This implies that, 4 families were recruited in the North (Makeni), 4 in the South (Bo), 4 in the East (Kenema), and 8 in the Western Area (Freetown). A Sample representation and demographic information of families, who participated in the face-to-face semi-structured interviews, are presented in the Table 1. A total of 20 families (20 husbands and 20 wives), a sample size of 40 , from various households were contacted across the country with a vivid explanation given to them about the study including potential risks of data publication, benefits to the country generally, and the assurance of confidentiality. The main participants in the study were husbands and wives (married couples) from different ethnic and religious backgrounds. The researcher ensured that an equal religious representation was selected for the interviews with ten families from each of the denominations (Muslim and Christian). The husbands and wives were interviewed separately to avoid any biasness or to prevent one couple influencing the other. Consequently, twenty families (20 husbands and 20 wives) were interviewed with $50 \%$ from each denomination (Muslim and Christian). Initial appointments and participant invitation letters; the research themes to be covered; and the participant information sheet detailing the interview protocol, commitment, benefits; and risks and confidentiality were issued to the interviewees at their various places of work before the official scheduled interviews at their homes.

\section{Interviews}

A guideline was developed for the entire research process, which was followed from the planning phase onto the implementation phase of the research to avoid any incongruity in the research process. The analysis of literature, guided the identification of theories and ideas that were tested using the data collected from the field. This was done in the form of a gap analysis. The researcher used open-ended questions and themes, from which a broad conclusion was drawn. The themes included Age, gender, associates/social relations, decision-making, ethnic group, friends/neighbours/family members, family image, extended family, identity. The interview for each respondent was scheduled for an hour, but on the average, it lasted between 50 and 55 minutes. The researcher carried out the interviews at the homes of the interviewees with the conversations recorded on a digital audio recorder.

\section{Data analysis}

The researcher transcribed all the data verbatim and imported them into NVIVO 10 to facilitate the analysis and coding. An iterative approach of reading and rereading the transcripts, identifying themes and patterns, and comparing across the data was used in analyzing the data. Thus, continuity in the coding process helped identify redundancies and overlaps in the categorization of the scheme, and then grouped both sequentially and thematically. The use of NVIVO 10 facilitated 
Table 1: Sample representation and demographic information of families who participated in the face-to-face semi-structured interviews.

\begin{tabular}{|c|c|c|c|}
\hline Families & & & Demographic \\
\hline \multirow{8}{*}{ Family 001} & \multirow{4}{*}{ Wife } & Location: HQ01 & \multirow{2}{*}{ Type of occupation: procurement office } \\
\hline & & Ethnicity: Creole & \\
\hline & & Family size: 3 & \multirow{2}{*}{ District/Provincial headquarter town: WA } \\
\hline & & Religion: Christianity & \\
\hline & \multirow{4}{*}{ Husband } & Location: HQ01 & \multirow{2}{*}{ Type of occupation: Banker } \\
\hline & & Ethnicity: Mende & \\
\hline & & Family size: 3 & \multirow{2}{*}{ District/Provincial headquarter town: WA } \\
\hline & & Religion: Christianity & \\
\hline \multirow{8}{*}{ Family 002} & \multirow{4}{*}{ Wife } & Location: HQ03 & 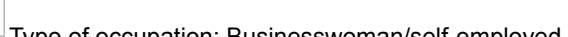 \\
\hline & & Ethnicity: Temne & lype of occupation: Businesswoman/selt-employed \\
\hline & & Family size: 8 & District/Provincial headquarter town NP \\
\hline & & Religion: Muslim & 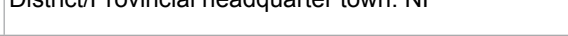 \\
\hline & \multirow{4}{*}{ Husband } & Location: HQ03 & \multirow{2}{*}{ Type of occupation: Constructor } \\
\hline & & Ethnicity: Temne & \\
\hline & & Family size: 8 & \\
\hline & & Religion: Muslim & District/Provinclal headquarter town: NP \\
\hline \multirow{8}{*}{ Family 003} & \multirow{4}{*}{ Wife } & Location: HQ04 & \multirow{2}{*}{ Type of occupation: Nurse } \\
\hline & & Ethnicity: Yalunka & \\
\hline & & Family size: 12 & \\
\hline & & Religion: Christianity & District/Provinclal neadquarter town: EP \\
\hline & \multirow{4}{*}{ Husband } & Location: HQ04 & \\
\hline & & Ethnicity: Kono & Type of occupation: Teacher \\
\hline & & Ethnicity: Yalunka & District/Provincial headquarter town EP \\
\hline & & Family size: 12 & 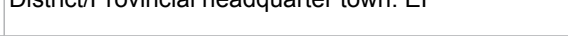 \\
\hline & & Location: HQ04 & Type of occupation: Geologist \\
\hline & Wife & Ethnicity: Temne & 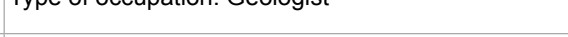 \\
\hline & Vurie & Family size: 7 & \\
\hline Family 004 & & Religion: Muslim & District/Provinclal headquarter town: EP \\
\hline 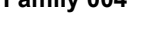 & & Location: HQ04 & Tyne of occunation: Banker \\
\hline & & Ethnicity: Mende & 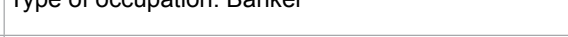 \\
\hline & 19 & Family size: 7 & District/Provincial headquarter town. FP \\
\hline & & Religion: Christianity & प \\
\hline & & Location: HQ02 & \\
\hline & Wife & Ethnicity: Mende & Type of occupation: Businesswoman/self-employed \\
\hline & Wuie & Family size: 5 & District/Provincial headquarter town: SP \\
\hline Familv 005 & & Religion: Christianity & 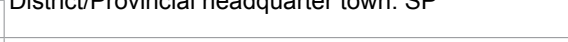 \\
\hline raminy 000 & & Location: HQ02 & Type of occupation: Police Officer \\
\hline & Hushand & Ethnicity: Mende & Type or occupation: Police Umicer \\
\hline & MUSDdna & Family size: 5 & District/Provincial headquarter town. SP \\
\hline & & Religion: Christianity & District/Provinclal headquarter town: SP \\
\hline & & Location: HQ02 & Type of occupation: Teacher \\
\hline & Wife & Ethnicity: Kono & Type or occupalion. Teacrer \\
\hline & Wvile & Family size: 5 & District/Provincial headquarter town SP \\
\hline & & Religion: Christianity & Districu//rovinclal neadquarter town: SP \\
\hline ramily 000 & & Location: HQ02 & \\
\hline & Husband & Ethnicity: Kissy & Type of occupation: Civil servant \\
\hline & T) & Family size: 5 & District/Provincial headdunarter town. SP \\
\hline & & Religion: Christianity & 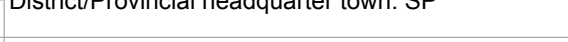 \\
\hline & & Location: HQ02 & Type of occupation: Businesswoman/self-employed \\
\hline & Wife & Ethnicity: Temne & \\
\hline & 然 & Family size: 4 & \\
\hline Family 007 & & Religion: Christianity & District/Provincial headquarter town: SP \\
\hline Family 000 & & Location: HQ02 & Type of occupation: Finance Officer \\
\hline & Husband & Ethnicity: Kono & yype of occupation. Finanice Umicer \\
\hline & Tusodilu & Family size: 4 & District/Provincial headquarter town SP \\
\hline & & Religion: Christianity & Districu//rovinclal neadquarter town: SP \\
\hline & & Location: HQ02 & Tyne of occunation. Social worker \\
\hline & Wife & Ethnicity: Mende & lype of occupation: soclal worker \\
\hline & Vuile & Family size: 10 & District/Provincial headquarter town: SP \\
\hline Familv 008 & & Religion: Christianity & 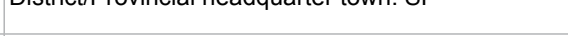 \\
\hline ramily 000 & & Location: HQ02 & Tyne of occunation: Social worker \\
\hline & Hushand & Ethnicity: Mende & Type or occupation: Soclal worker \\
\hline & Thusintu & Family size: 10 & \\
\hline & & Religion: Christianity & District/Provincial headquarter town: SP \\
\hline
\end{tabular}


Citation: Kakay S. An analysis of the impact of reference groups on collectivist families'meal social interaction behaviour in Sierra Leone. J pub health catalog. 2018;1(2):41-52.

\begin{tabular}{|c|c|c|c|}
\hline \multirow{8}{*}{ Family 009} & \multirow{4}{*}{ Wife } & Location: HQ03 & \multirow{2}{*}{ Type of occupation: mid-wife } \\
\hline & & Ethnicity: Temne & \\
\hline & & Family size: 4 & \multirow{2}{*}{ District/Provincial headquarter town: SP } \\
\hline & & Religion: Muslim & \\
\hline & \multirow{4}{*}{ Husband } & Location: HQ03 & Tune of ocunotion. Rucino comon/colf omploued \\
\hline & & Ethnicity: Madingo & Iype or occupauon. Businessman/sen-empioyed \\
\hline & & Family size: 4 & Dictrict/Provincial headauarter town. NP \\
\hline & & Religion: Muslim & Uistrict/Provinclal neadquarter town: NP \\
\hline \multirow{8}{*}{ Family 010} & \multirow{4}{*}{ Wife } & Location: HQ03 & \multirow{2}{*}{ Type of occupation: Teacher } \\
\hline & & Ethnicity: Koranko & \\
\hline & & Family size: 10 & \\
\hline & & Religion: Muslim & District/Provincial headquarter town: NP \\
\hline & \multirow{4}{*}{ Husband } & Location: HQ03 & \multirow{2}{*}{ Type of occupation: Civil engineer } \\
\hline & & Ethnicity: Mende & \\
\hline & & Family size: 10 & \multirow{2}{*}{ District/Provincial headquarter town: NP } \\
\hline & & Religion: Muslim & \\
\hline \multirow{8}{*}{ Family 011} & \multirow{4}{*}{ Wife } & Location: HQ01 & Tune of onoungtion Tonobor \\
\hline & & Ethnicity: Mende & lype of occupation: leacher \\
\hline & & Family size: 8 & Dictrict/Provincial headauarter toun. W/A \\
\hline & & Religion: Muslim & 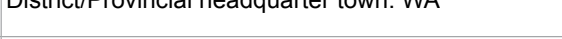 \\
\hline & \multirow{4}{*}{ Husband } & Location: HQ01 & Tyne of occunation. Civil cervant \\
\hline & & Ethnicity: Mende & lype of occupatıon: Civil servant \\
\hline & & Family size: 8 & District/Provincial headruarter town. W/A \\
\hline & & Religion: Muslim & District/Provinclal neadquarter town: VVA \\
\hline \multirow{8}{*}{ Family 012} & & Location: HQ01 & Tyne of occunation. Teacher/Pastor \\
\hline & & Ethnicity: Limba & Tyре от оccupation: Teacner/rastor \\
\hline & VVITe & Family size: 6 & District/Provincial headguarter town :WA \\
\hline & & Religion: Christianity & District/Provincial headquarter town: VVA \\
\hline & & Location: HQ01 & \\
\hline & & Ethnicity: Mende & Type of occupation: Lecturer \\
\hline & Husband & Family size: 6 & \\
\hline & & Religion: Christianity & District/Provincial headquarter town: VVA \\
\hline & & Location: HQ04 & \\
\hline & & Ethnicity: Creole & Type of occupation: Nurse \\
\hline & Wite & Family size: 4 & District/Provincial headauarter town EP \\
\hline Family 013 & & Religion: Muslim & District/Provinclal neadquarter town: EP \\
\hline ramily 013 & & Location: HQ04 & Tyne of occunation. I ecturer \\
\hline & & Ethnicity: Limba & \\
\hline & Husband & Family size: 4 & District/Provincial headauarter town : EP \\
\hline & & Religion: Muslim & District/Provincial headquarter town: EP \\
\hline & & Location: HQ03 & Tyne of occunation . Teacher \\
\hline & Wife & Ethnicity: Temne & lype of occupation: leacher \\
\hline & Vite & Family size: 5 & District/Provincial headquarter town: NP \\
\hline & & Religion: Christianity & District/Provincial headquarter town: NP \\
\hline Family 014 & & Location: HQ03 & \\
\hline & & Ethnicity: Temne & Type of occupation: Agricultural Officer \\
\hline & Husband & Family size: 5 & \\
\hline & & Religion: Christianity & District/Provincial headquarter town: NP3 \\
\hline & & Location: HQ01 & Tyne of occunation. Univercity Adminictrator \\
\hline & Whifo & Ethnicity: Creole & Iype of occupation: Unıversity Admınıstrator \\
\hline & VVITe & Family size: 4 & District/Provincial headguarter town . W/A \\
\hline Familv 015 & & Religion: Christianity & District/Provincial headquarter town: WA \\
\hline & & Location: HQ01 & \\
\hline & Hushand & Ethnicity: Creole & Type of occupation: University Administrator \\
\hline & Husband & Family size: 4 & District/Provincial headguarter town W WA \\
\hline & & Religion: Christianity & District/Provinclal headquarter town: VWA \\
\hline & & Location: HQ01 & \\
\hline & & Ethnicity: Mende & Type of occupation: Principal \\
\hline & Vite & Family size: 8 & \\
\hline Family 016 & & Religion: Muslim & District/Provincial headquarter town: WA \\
\hline Family vio & & Location: HQ01 & \\
\hline & & Ethnicity:Yalunka & Type of occupation: Deputy Director (Civil Servant) \\
\hline & Husband & Family size: 8 & Dictrict/Provincial headguarter town w/A \\
\hline & & Religion: Muslim & District/Provinclal neadquarter town: VVA \\
\hline
\end{tabular}




\begin{tabular}{|c|c|c|c|}
\hline \multirow{8}{*}{ Family 017} & \multirow{4}{*}{ Wife } & Location: HQ01 & \multirow{2}{*}{ Type of occupation: Businesswoman/Self-employed } \\
\hline & & Ethnicity: Temne & \\
\hline & & Family size: 9 & \multirow{2}{*}{ District/Provincial headquarter town: WA } \\
\hline & & Religion: Muslim & \\
\hline & \multirow{4}{*}{ Husband } & Location: HQ01 & \multirow{2}{*}{ Type of occupation: Medical Lecturer } \\
\hline & & Ethnicity: Fullah & \\
\hline & & Family size: 8 & \multirow{2}{*}{ District/Provincial headquarter town: WA } \\
\hline & & Religion: Muslim & \\
\hline \multirow{8}{*}{ Family 018} & \multirow{4}{*}{ Wife } & Location: HQ01 & \multirow{2}{*}{ Type of occupation: Teacher } \\
\hline & & Ethnicity: Mende & \\
\hline & & Family size: 8 & \multirow{2}{*}{ District/Provincial headquarter town: WA } \\
\hline & & Religion: Muslim & \\
\hline & \multirow{4}{*}{ Husband } & Location: HQ01 & Tyment \\
\hline & & Ethnicity: Madingo & Type of occupation: Businessman/self-employed \\
\hline & & Family size: 8 & Dictrict/Provincial headaunter town W W \\
\hline & & Religion: Muslim & District/Provinclal neadquarter town: WA \\
\hline \multirow{8}{*}{ Family 019} & \multirow{4}{*}{ Wife } & Location: HQ01 & \multirow{2}{*}{ Type of occupation: Housewife } \\
\hline & & Ethnicity: Mende & \\
\hline & & Family size: 12 & \\
\hline & & Religion: Muslim & District/Provincial headquarter town: WA \\
\hline & \multirow{4}{*}{ Husband } & Location: HQ01 & \multirow{2}{*}{ Type of occupation: Civil servant (Deputy Director General) } \\
\hline & & Ethnicity: Mende & \\
\hline & & Family size: 12 & \multirow{2}{*}{ District/Provincial headquarter town: WA } \\
\hline & & Religion: Muslim & \\
\hline \multirow{8}{*}{ Family 020} & \multirow{4}{*}{ Wife } & Location: HQ04 & \multirow{2}{*}{ Type of occupation: Social worker } \\
\hline & & Ethnicity: Mende & \\
\hline & & Family size: 3 & \multirow{2}{*}{ District/Provincial headquarter town: EP } \\
\hline & & Religion: Muslim & \\
\hline & \multirow{4}{*}{ Husband } & Location: HQ04 & \multirow{2}{*}{ Type of occupation: Social worker } \\
\hline & & Ethnicity: Madingo & \\
\hline & & Family size: 3 & \multirow{2}{*}{ District/Provincial headquarter town: EP } \\
\hline & & Religion: Muslim & \\
\hline
\end{tabular}

Note: Freetown: HQ01; Western Area: WA; Bo: HQ02; Southern province: SP; Makeni: HQ03; Northern Province: NP; Kenema: HQ04; Eastern province: $\mathbf{E P}$. Please note that, $\boldsymbol{H Q}$ means-Headquarter town.

the development of an audit trail through the use of memos, providing evidence of confirmation of the research findings. After collating and coding, the data was summarized and organized by comparing the responses provided by the different family (husband and wife) members, and conceptualized the interpretation of each category by each family member, and how they interact with each other. The researcher noted that sometimes, there were variations in responses from different family members, which could have prompted the use of more than one code, which resulted in the building up of different sub-categories. The researcher worked on the categorization scheme, assignment of codes, and interpreted and reviewed the transcripts independently. Where there were differences in interpretations, commonalities and differences were identified and interpreted appropriately. Therefore, the researcher used triangulation to enhance the credibility of the data. Also, the audio-recordings and associated transcripts (field notes) were transcribed as soon as the researcher returned from the field to avoid unnecessary build-up of information and data and avoid loss of vital information.

\section{Results}

The researcher used a sample of 40 respondents, who were between the ages of 18 and 65 years, as participants in the one-to-one semi-structured face-to-face interview. A tabular representation of the sample and personal data of the respondents are depicted in Table 1 . The researcher considered the husband and wife (married couples) in each family as the main participants in the interview process. About twenty families (20 husbands and 20 wives) were selected in order to get a balanced response and interpretation of the results, and to reduce biasness to the bare minimum. A tabular summary of the degree of predominance of the reference group factors that influenced families' meal social interaction behaviour are depicted in Table 2. It was imperative that, after the twentieth family, the data was saturated as the information collected from the $18^{\text {th }}, 19^{\text {th }}$ and $20^{\text {th }}$ families $\left(35^{\text {th }}, 36^{\text {th }}, 37^{\text {th }}, 38^{\text {th }}, 39^{\text {th }}\right.$ and $40^{\text {th }}$ interviewees) were similar to those stated by earlier respondents.

\section{Key findings of the study}

The analysis of this study identified a number of themes and sub-themes, as key reference group influencers of families' meal social interaction behaviour, including age, gender, decision-making, associates, extended family and identity. A comprehensive evaluation and discussion of the influence of each sub-groups on participating males and females was undertaken. The themes and sub-themes that emerged from the study are depicted in the Table 3 and the Figure 1 below:

\section{The impact of Age on families' meal social interaction} behaviour

Multiple female participants (Muslim and Christian) highlighted respect as fundamental reference group attributes that influences their families' meal social interaction behaviour. This suggests that age is an essential ingredient for determining the degree of involvement in social interaction at mealtimes, 
Citation: Kakay S. An analysis of the impact of reference groups on collectivist families'meal social interaction behaviour in Sierra Leone. J pub health catalog. 2018;1(2):41-52.

Table 2: A Summary of the reference groups factors that influenced families'meal social interaction behaviour.

\begin{tabular}{|c|c|c|}
\hline \multicolumn{3}{|c|}{ Reference Groups } \\
\hline \multirow{5}{*}{ Age } & Respect & Predominant in all families \\
\hline & Hierarchy & Predominant in all families \\
\hline & Wisdom & Emphasised by a majority (CM, MM, MF), but less by $\mathrm{CF}$ \\
\hline & Authority/guidance & Predominant in all families \\
\hline & Experience & Emphasised by a majority of Muslims only (MM, MF) \\
\hline \multirow{2}{*}{ Gender } & No impact & Predominant in all families \\
\hline & Gender distinction & Predominant in all families \\
\hline \multirow{3}{*}{ Associates } & Idea sharing & Emphasised by a majority (CM, CF, MF), but less by MM \\
\hline & Regulation/control & Emphasised by a majority of females regardless of religion ( $\mathrm{CF}, \mathrm{MF}$ ) \\
\hline & Building relationship & Predominant in all families \\
\hline \multirow{4}{*}{ Decision-making } & Planning & Predominant in all families \\
\hline & Authority/hierarchy/guidance/control & Emphasised by a majority (CM, CF, MM), but less by MF \\
\hline & Development & Emphasised by a majority of MF only \\
\hline & Family unity/stability & Emphasised by a majority of females regardless of religion (CF, MF) \\
\hline \multirow{3}{*}{ Extended families } & Background differences & Predominant in all families \\
\hline & Economic/financial constraints & Emphasised by a majority (CF, MM, MF), but less by $\mathrm{CM}$ \\
\hline & Domestic chores & Emphasised by a majority of Muslims only (MM,MF) \\
\hline Identity & Position/rank & Predominant in all families \\
\hline
\end{tabular}

Table 3: Thematic Analysis and schematic summary diagrams of the impact of reference groups on families 'meal social interaction behaviour.

\begin{tabular}{|c|c|c|c|}
\hline \multicolumn{4}{|r|}{ Interviews with Families } \\
\hline Literature & Questions & Field themes & Field sub-themes \\
\hline \multirow{17}{*}{$\begin{array}{l}\text { Reference } \\
\text { Groups }\end{array}$} & \multirow{3}{*}{$\begin{array}{l}\text { How important is age } \\
\text { in your family's social } \\
\text { interaction at the } \\
\text { dinner table? }\end{array}$} & Hierarchy & $\begin{array}{l}\text { Position, ranking, expectations, respect (MW, } \mathrm{CW}, \mathrm{MH}, \mathrm{CH}) \text {, obedience, roles, maturity, responsibility, assistance, } \\
\text { contribution }(\mathrm{MH}) \text {, Preference, seniority, maturity }(\mathrm{CW}) \text {, family head, elderliness, hierarchy (MW, } \mathrm{CW}, \mathrm{MH}, \mathrm{CH})\end{array}$ \\
\hline & & Authority & $\begin{array}{l}\text { Shapes behaviour, control behaviour, boundaries, peace/harmony }(\mathrm{MW}, \mathrm{MH}) \text {, command, love }(\mathrm{CW}) \text {, protection } \\
(\mathrm{CW}, \mathrm{MH}) \text {, rules, regulations, understanding/tolerance }(\mathrm{CW}, \mathrm{MH}) \text {, unity/stability }(\mathrm{MH}) \text {, authority }(\mathrm{MW}, \mathrm{CW}, \mathrm{MH} \text {, } \\
\mathrm{CH}) \text {, guidance }\end{array}$ \\
\hline & & $\begin{array}{l}\text { Decision- } \\
\text { making }\end{array}$ & $\begin{array}{l}\text { Wisdom }(\mathrm{MW}, \mathrm{CW}, \mathrm{MH}, \mathrm{CH}) \text {, experience }(\mathrm{MW}, \mathrm{CW}, \mathrm{MH}) \text {, advice }(\mathrm{MW}, \mathrm{MH}) \text {, information /communication }(\mathrm{MH}) \text {, } \\
\text { responsibility, learning }(\mathrm{MW}) \text {, commonality, direction, contentment }(\mathrm{MH}) \text {, success }(\mathrm{MH}) \text {, decision-making }(\mathrm{MW} \text {, } \\
\mathrm{MH}, \mathrm{CH}) \text {, blessing }(\mathrm{MH}) \text {, values }(\mathrm{CH}) \text {, affection }(\mathrm{CH}) \text {, participation }(\mathrm{CH})\end{array}$ \\
\hline & \multirow{2}{*}{$\begin{array}{l}\text { Why does gender } \\
\text { influence the way your } \\
\text { family interact socially } \\
\text { at the dinner table? }\end{array}$} & Task distribution & Domestic chores, role definition ( $\mathrm{MW}, \mathrm{MH}, \mathrm{CH})$, responsibility, food quality $(\mathrm{CH})$ \\
\hline & & $\begin{array}{l}\text { Gender } \\
\text { distinction }\end{array}$ & $\begin{array}{l}\text { Eating boundaries }(\mathrm{MW}, \mathrm{CW}) \text {, position, breadwinner }(\mathrm{MW}) \text {, food quality, respect, food preparation, male } \\
\text { supremacy, }(\mathrm{MW}) \text {, learning }(\mathrm{MW}) \text {, gender distinction }(\mathrm{MH}, \mathrm{CH}) \text {, gender separation }(\mathrm{MH}, \mathrm{CH}) \text {, authority }(\mathrm{MH}, \mathrm{CH}) \text {, } \\
\text { respect }(\mathrm{CW}, \mathrm{MH})\end{array}$ \\
\hline & \multirow{4}{*}{$\begin{array}{l}\text { In what ways does } \\
\text { the importance of } \\
\text { associates influence } \\
\text { your family's } \\
\text { interaction at the } \\
\text { dinner table? }\end{array}$} & $\begin{array}{l}\text { Control/ } \\
\text { regulation }\end{array}$ & $\begin{array}{l}\text { Guidance }(\mathrm{CH}) \text {, correct behaviour, direction, shape behaviours, behaviour improvement, rules, identity }(\mathrm{MH}) \text {, } \\
\text { politeness }(\mathrm{MH}) \text {, control/regulation }(\mathrm{MW}, \mathrm{CW}, \mathrm{MH}) \text {, respect }(\mathrm{MW}, \mathrm{CW}, \mathrm{MH})\end{array}$ \\
\hline & & Education & $\begin{array}{l}\text { Solution, cultural learning, social etiquette, advice }(\mathrm{MW}, \mathrm{CH}) \text {, idea sharing/education }(\mathrm{MW}, \mathrm{CW}, \mathrm{MH}, \mathrm{CH}) \text {, civility } \\
(\mathrm{CW}) \text {, table manners, idea generation, values }(\mathrm{CH}) \text {, beliefs, social learning, family history }(\mathrm{MH}) \text {, knowledge } \\
\text { sharing, training, troubleshooting }(\mathrm{MW}, \mathrm{CH})\end{array}$ \\
\hline & & Affection & $\begin{array}{l}\text { Understanding, bonding, love/affection (MW, CW, MH), care, social relationship/building relationship (MW, CW, } \\
\mathrm{MH}, \mathrm{CH}) \text {, unity/stability ( } \mathrm{MW}, \mathrm{CW}, \mathrm{CH}) \text {, closeness, togetherness, happiness, help, trust, meal sharing (MW, } \\
\mathrm{MH}) \text {, socialization }(\mathrm{CH}) \text {, connectivity, family image }(\mathrm{MH}) \text {, compassion, oneness, sympathy, cordiality, sense of } \\
\text { belonging, prayer }(\mathrm{CW}) \text {, expectation }(\mathrm{CW}) \text {, blessing }(\mathrm{CW}) \text {, participation }(\mathrm{CH})\end{array}$ \\
\hline & & $\begin{array}{l}\text { Social/economic } \\
\text { costs }\end{array}$ & "Resource overburden (MW, MH), conflict (MW, CW), thieving (CW), anti-social behaviour" \\
\hline & \multirow{3}{*}{$\begin{array}{l}\text { How important is } \\
\text { decision-making in } \\
\text { your family's social } \\
\text { interaction at the } \\
\text { dinner table? }\end{array}$} & Planning & $\begin{array}{l}\text { Guidance, direction }(\mathrm{CW}, \mathrm{MH}) \text {, family interest, development }(\mathrm{MW}, \mathrm{CW}, \mathrm{MH}, \mathrm{CH}) \text {, right path, solution, growth, } \\
\text { prioritisation }(\mathrm{MW}) \text {, idea generation, character moulding, responsibility, family safety, consultation (MW, } \mathrm{CW} \text {, } \\
\mathrm{MH}, \mathrm{CH}) \text {,learning }(\mathrm{MH}, \mathrm{CH}) \text {, reduce wastage }(\mathrm{MH}) \text {, consent }(\mathrm{MH}) \text {, achievement }(\mathrm{MH}) \text {, choices }(\mathrm{MH}) \text {, right track, } \\
\text { organising }(\mathrm{MW}, \mathrm{MH}), \text { management/coordination }(\mathrm{MH}) \text {, view sharing, prosperity/progress/success }(\mathrm{MW}, \mathrm{CW}) \text {, } \\
\text { rationality }(\mathrm{CH}) \text {, projection, conscientiousness, judgement }(\mathrm{CH}) \text {, planning }(\mathrm{MW}, \mathrm{CW}, \mathrm{MH}, \mathrm{CH}) \text {, task distribution } \\
(\mathrm{CW}) \text {, collaboration }(\mathrm{CH})\end{array}$ \\
\hline & & Family cohesion & $\begin{array}{l}\text { Unity/cohesion/stability }(\mathrm{MW}, \mathrm{CW}, \mathrm{CH}) \text {, peace/tranquility }(\mathrm{MW}) \text {, happiness, love, understanding }(\mathrm{MH}, \mathrm{CH}) \text {, } \\
\text { relationship building, harmony }(\mathrm{CW}, \mathrm{MH}) \text {, equality }(\mathrm{MH}) \text {, fairness, togetherness, socialisation, collaboration, } \\
\text { collectiveness, care }(\mathrm{MW}) \text {, participation }(\mathrm{CW}) \text {, family image }(\mathrm{MH})\end{array}$ \\
\hline & & $\begin{array}{l}\text { Hierarchy/ } \\
\text { authority }\end{array}$ & $\begin{array}{l}\text { Respect }(\mathrm{MW}) \text {, shape behaviour, authority/control/guidance }(\mathrm{MW}, \mathrm{CW}, \mathrm{MH}, \mathrm{CH}) \text {, maintain order, age }(\mathrm{MW}) \text {, seniority, } \\
\text { role definition, discipline }(\mathrm{CW}) \text {, orderliness, correct behaviour, rules, obedience }(\mathrm{MH}, \mathrm{CH}) \text {, family head, command, } \\
\text { dictatorship, listening, regulation, contribution }(\mathrm{CH}) \text {, responsibility }(\mathrm{CW}) \text {, safeguards }(\mathrm{CW}) \text {, democracy }(\mathrm{CH})\end{array}$ \\
\hline & \multirow{3}{*}{$\begin{array}{l}\text { How do extended } \\
\text { family members affect } \\
\text { your family's meal } \\
\text { social interaction } \\
\text { behaviour? }\end{array}$} & Economic costs & Economic and financial constraints (MW, CW, MH), Resource, expenditure, food quantity, food quality \\
\hline & & Social costs & $\begin{array}{l}\text { Gossip, different background (MW, } \mathrm{CW}, \mathrm{MH}, \mathrm{CH}) \text {, cultural variation, divulge family secret }(\mathrm{MW}, \mathrm{CW}, \mathrm{MH}) \text {, breed } \\
\text { hatred }(\mathrm{MW}, \mathrm{CW}, \mathrm{MH}) \text {, stealing/thieving }(\mathrm{MW}, \mathrm{CW}, \mathrm{MH}, \mathrm{CH}) \text {, witchcraft/evil spirit }(\mathrm{MW}, \mathrm{MH}) \text {, quarrels, conflict/ } \\
\text { misunderstanding }(\mathrm{MW}, \mathrm{CW}, \mathrm{MH}, \mathrm{CH}) \text {, envy, feud, animosity, jealousy/comparison }(\mathrm{MW}, \mathrm{CW}, \mathrm{MH}, \mathrm{CH}) \text {, stalls } \\
\text { development }(\mathrm{MH}, \mathrm{CH}) \text {, ostracisation }(\mathrm{MH}) \text {, bullying }(\mathrm{CH}) \text {, obligatory }(\mathrm{MH}, \mathrm{CH}) \text {, misunderstanding, backbiting/ } \\
\text { gossip }(\mathrm{MW}, \mathrm{CW}, \mathrm{MH}) \text {, behavioural challenges }(\mathrm{MH}) \text {, malice }(\mathrm{MH})\end{array}$ \\
\hline & & Social benefits & $\begin{array}{l}\text { Sharing }(\mathrm{MW}, \mathrm{CW}, \mathrm{MH}) \text {, domestic chores }(\mathrm{MW}, \mathrm{CW}, \mathrm{MH}, \mathrm{CH}) \text {, advice }(\mathrm{MW}) \text {, shape behaviour, lineage knowledge } \\
(\mathrm{CW}) \text {, bonding }(\mathrm{MH}) \text {, reduce workload, Domino effect }(\mathrm{MH}, \mathrm{CH}) \text {, love, share ideas, cordiality, subsidise income } \\
(\mathrm{MH}) \text {, appreciation, gifts }(\mathrm{CH}) \text {, gratification/gratification }(\mathrm{CH}) \text {, charitable act }(\mathrm{MH}) \text {, cultural beliefs }(\mathrm{MH})\end{array}$ \\
\hline & \multirow{2}{*}{$\begin{array}{l}\text { What is your opinion of } \\
\text { the definition of identity } \\
\text { within the family when } \\
\text { interacting at the } \\
\text { dinner table? }\end{array}$} & $\begin{array}{l}\text { Authority/ } \\
\text { hierarchy }\end{array}$ & $\begin{array}{l}\text { Position/rank (MW, CW, } \mathrm{MH}, \mathrm{CH}) \text {, control }(\mathrm{MW}, \mathrm{CW}, \mathrm{MH}) \text {, respect }(\mathrm{MW}, \mathrm{MH}, \mathrm{CH}) \text {, roles/responsibilities }(\mathrm{MW} \text {, } \\
\mathrm{MH}, \mathrm{CH}) \text {, boundaries, seniority, shape behaviour, priority }(\mathrm{MH}) \text {, age }(\mathrm{MH}) \text {, headship, obedience }(\mathrm{MH}) \text {, guidance, } \\
\text { Orderliness }(\mathrm{CH}) \text {, law and order, right and wrong }(\mathrm{CW})\end{array}$ \\
\hline & & Family cohesion & $\begin{array}{l}\text { Discrimination (MW, CH), togetherness/unity }(\mathrm{CW}) \text {, relationship definition, sense of belonging }(\mathrm{CW}) \text {, love, } \\
\text { distinction/differentiation }(\mathrm{CW}) \text {, discord, learning }(\mathrm{CH}) \text {, expectations }(\mathrm{MW}) \text {, boundaries }(\mathrm{CW})\end{array}$ \\
\hline
\end{tabular}




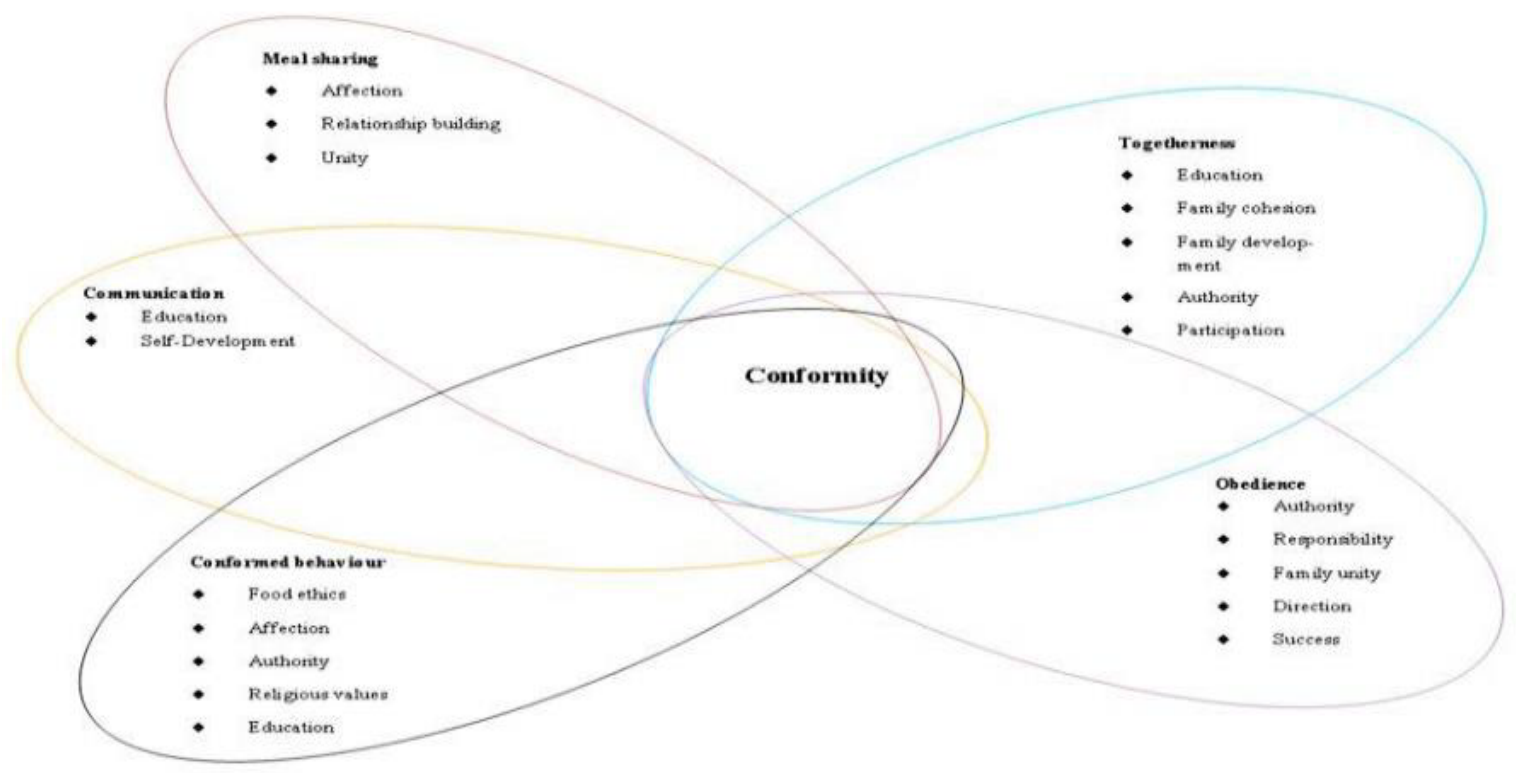

Figure 1: The effect of reference groups on collectivist families'meal behaviour.

as children should not be involved in adult discussion, as it symbolizes disrespect. Consequently, it has the resultant effect in determining the order of food distribution, and to some extent the quantity of food access by individuals at mealtimes.

"Age is very important as it serves authority and determinant of whom to respect and who not to talk freely to at the dinner table. When an elder is speaking to you at the dinner table, it is important you abide by his/her command. If I am not around, the eldest daughter/son takes responsibility for the entire family and the younger ones must respect her and the orders she gives. Age enables us to determine who is older than the other and determines who should be respected at the dinner table because you cannot ask the elder one to fetch water when the younger is seated at the same table" (Interviewee 5, Female, Christian)

However, Muslim females highlighted the symbolism of authority as fundamental arbiter of age, which was less emphasized by Christian females. They argued that, age define the roles and responsibilities of individuals in a family, and acts as a source of wisdom and experience. This implies that, age is an instrument/tool used by adults to provide appropriate advice and guidance to children at the dinner table, which enables them to discern between rights and wrongs.

"Age is important as it signifies respect, authority, wisdom and experience. In our culture, it is always important to give respect to your elders, whether they are your parents or not. At the dinner table, children are not allowed to talk in the presence of elders as a symbol of respect". (Interviewee 21, Female, Muslim)

In tandem with the females, an overwhelming number of male participants (Muslim and Christian) identified respect, as critical to their families' meal social interaction behaviour. They professed that, as a mark of respect, children should not have eye contact with adults at mealtimes, but to concentrate and focus on the food in front of them. It is also fundamentally the responsibility of young ones to hold the dish/plate/bowl in position when eating from a common dish/plate/bowl with elders, and they are responsible for fetching water for washing hands before eating. Adherence to this responsibility, they claimed, can help guide and protect children from making mistakes and pushes them to behave properly in the public.

"Age of course depicts the hierarchy of one's family and because you are either the father, mother, eldest son/daughter, it is onerous on everybody, especially at the dinner table to respect people that are older than you. In Africa, we respect age a lot and as a family it is paramount that we give credence to age, particularly to people that are older than you. So age is very important as it signifies authority and who should do what in the home" (Interviewee 14, Male, Christian)

\section{The impact of gender on families' meal social interaction behaviour}

Role distinction and gender separation at mealtimes emerged as a primary determinant of families' meal social interaction behaviour. However, the degree of influence varies between the Muslim and Christian females, as the practice is predominant among Muslim females than their Christian counterparts. Participants who embrace the practice argued that, females are socialized to perform the traditional role of cooking, serving, and cleaning the dining area after eating; whilst males are socialized to perform the role of breadwinners and/or heads of the families. It is fundamentally a normal traditional practice in some families, for males and females to eat separately in groups, as males are socialized and trained to assume leadership roles, whilst females assume subservient roles.

"Well, in my family, we most times eat from a common bowl, and women are only allowed to eat together with women and men are also expected to eat with men. Also, greater attention is given to male children than the female ones because my husband always sees the male children as his future inheritance. He always signals that the women will one day get married and absorbed into another family while the men will always bear his 
name. So at the dinner table, he gives the male children more attention than the females. Also, the cleaning of the pots, pans and plates are normally done by the females and they are also required to sweep and clean the dinner table before and after eating". (Interviewee 33, Female, Muslim)

"Well, no, gender has no significance when we are interacting at the dinner table. We have gender equity. We don't discriminate between our children to say that this is a girl or this is a boy and that they should behave in a specific way at the dinner table". (Interviewee 15, Female, Christian)

Despite the prevalence of the practice across different ethnic and religious groups, many Muslim and Christian males emphasized that gender has limited impact on their families' socialization at mealtimes. This is largely due to their exposure to western education and urban acculturation. It is imperative that, the exposure of some families in the urban areas to Western education and urbanization has forced them to renege on traditional practices inherited from their forebears.

"Despite being a Muslim, but my level of education bars me from discriminating my children because I do not even know who will be what later in life. However, in my culture, we expect to women to sit and eat together as a unit and from a common bowl and the men are also expected to eat together from the same bowl. But I don't encourage that in my family, may be because of my level of education". (Interviewee 36, Male, Muslim)

\section{The impact of associates on families' meal social interaction behaviour}

Several Muslim and Christian females highlighted associates/ non-family members as significant influencers of their families' meal social interaction behaviour. Socializing with associates refines the behaviour of the children at mealtimes, as it teaches them how to love others, improves social etiquette, and foster better relation with outsiders. It also helps the children to cultivate the idea of sharing and teaches them the morals of life, as the African culture professed that, "no man is an Island. The perceived value of associates, as proclaimed by many participants, is that they help the family with their domestic chores. They have also been touted to settle family disputes, subsidize family incomes and act as a source of advice to the family. Despite the avowed acknowledgement of their positive impact on families, but a wind of caution advanced by a few participants is that, associates with bad behaviours/characters, including thieving and vulgarity can impact negative on the families' meal social interaction behaviour.

"Well, like I said earlier, we have a system of shared beliefs and values in my family, wherein we are always open to strangers sharing meal with us at the dinner table irrespective of the background of the visitor. We are always open...it teaches the children the importance of sharing, particularly in Sierra Leone were opportunities are scarce. When God provides for the family it is always important that we share with other people as this will act as blessing to the family...builds an everlasting bond of respect and trust as they will always say good things about your family outside...forum of sharing ideas..." (Interviewee 27, Female, Christian)
In tandem with the female participants, the male participants (Muslim and Christian) proclaimed that, associates creates an avenue for building relationship with neighbours, friends and other people in the community, which significantly increases the families' external influence and reach. Despite the commonality shared, several Christian males emphasized idea sharing and rich cultural education, as key attributes associates bring to the dinner table. They also argued that, associates provide a rich forum for discussion and idea sharing after meal. However, associates can also be a drain on families' resources, as unplanned visitors sometimes can limit the amount of food accessed by children, which makes them very unhappy after dinner.

"In the area of having friends, colleagues or relatives visiting, and having meal together, that teaches the children that they have to share. In some settings when a visitor comes unannounced and you meet them eating, they will give you a newspaper to read. I consider that as rather unfortunate and that you are teaching the children not to share and no man is an island. ... The kids will grow-up with that culture. ... Our culture pays great credence to sharing as it serves as the bond that ties people together-between you and your neighbour, between you and your classmates, between you and your relations. My children know that it is good to share and I am a firm believer in that. ... Imagine you are hungry and you see your friend eating, and all he could do is to give you a newspaper to read. ... However, having other nonfamily members partake in your meal occasionally, teaches the kids the culture of sharing and that is one way of expressing love and concern for one another". (Interviewee 28, Male, Christian)

\section{The impact of decision-making on families' meal social interaction behaviour}

Planning, as emphasized by several participants (Muslim and Christian females), is a critical decision-making factor, as it helps determine the food expenditure pattern of the family. It provides guidance as to how the family uses their resources, which is essential to their day-to-day survival and/or sustainability. They further argued that, authority in decision-making is fundamental to the success of the family, as it gives parents the ability to correct/stream-line their children's behaviours. This, they argued, help unify the family around a common purpose and reduce chaos at mealtimes. However, many Muslim female participants, unlike their Christian counterparts, emphasized that, decision-making enhances their families' stability, development and progress, as it is an instrument for fostering peaceful co-existence and respect in the family.

"Decision is very important in all the things we do as family from children's school fees discussed at the dinner table to the amount spent on feeding all needs to be decided by me and my husband and sometimes we even involve the children into it. Also, decision making can be a very good control instrument. For example, if my daughter misbehaves at the dinner table and we ignore it and fail to decide on the line of action to take to correct her behaviour, she will end up becoming a bad person... It is very important because like I said it is what keeps the family together. A family without a decision-maker can never be able to enjoy stability or to maintain order at the dinner table". (Interviewee 31, Female, Muslim)

Consistent with the views of the females, Muslim and Christian 
males also highlighted planning, authority and unity as essential decision-making ingredients that promotes their families' stability and progress, which help stream-line their children's behaviour at mealtimes.

"Decision-making in a family is very important. It is only through decision-making that we are able to plan for the future of the family. Sometimes we use decision to give the family direction and it is through decision-making that we are able to correct the wrongs of a child and make them right. If as a father I cannot make the right decision that will guide my family, they will all end up becoming very bad people in society". (Interviewee 36, Male, Muslim)

\section{The impact of extended family on families' meal social interaction behaviour}

Participants (Muslim and Christian females) described background difference, as key extended family issue that affect their meal social interaction behaviour. This difference makes it very difficult for appropriate cultural fit to occur between the family and their extended relations at mealtimes. The financial and economic burden that extended family brings to bear on families, were considered a crucial factor, as it determines the amount of food that is available to family members at mealtimes. This implicitly suggests that, many families have limited financial resources and therefore, cannot afford the additional burden that extended families bring to their dinner tables. However, unlike their Christian counterparts, Muslim female participants reiterated performing domestic chores, as the primary benefit extended families have on their families' meal behaviour.

"Like I said earlier, it is source of stealing in the home, particularly if the extended family members' character is different from those of your family. There will always be quarrels, backbiting and comparison between your children and the children of the extended family members, which breeds hatred and animosity... affects the way your family lives and they are also constraint to resources... also affect the level of expenditure in terms of the quantity of food to purchase for the family as well as the quality”. (Interviewee 27, Female, Christian)

"Well, sometimes extended family can be good, but some other times, they can be bad. Let me talk about the good first. If you have extended family members with good behaviour that can be reflected on your children and they also help with household chores. But if the extended family is one of bad character, they have the tendency of negatively influencing your children. Also, extended families can be a burden on resources, especially when things are not right at home". (Interviewee 21, Female, Muslim)

Invariably, the Muslim and Christian males professed that background differences significantly hinders cohesion with extended families, and this also hampers relationship at the dinner table. Participants were increasingly concerned about the debilitate effect the background differences is causing in their families, including thieving, backbiting (divulging families' internal secrets to outsiders), malice and quarrels, bad character/ behaviour and disobeying orders. Despite the shared similarity, it is indicative as suggested by several Muslim male participants that economic or financial constraints and assistance with domestic chores are major influences extended families have on their families' mealtime behaviours.

"It poses huge problems because since they are relations, it is very difficult to drive them from home irrespective of their behaviour or character. You will always be scared of what other members of your extended family will say or feel about you. For example, if the person that joined your family from one of your extended relation, say nephew/niece, is a thief, that will induce your own son/daughter to start imitating the same behaviour. That will greatly affect interaction in the family both at the dinner table and outside it". (Interviewee 6, Male, Christian)

"Extended family is a burden when you look at it from a financial point of view. There are also behavioural challenges associated with extended family. For example, the negative outside influence of the extended family normally feeds into the family, which may disrupt the smooth functioning of the family...The good aspects of extended family members is that if you have the right kind of person, that person will support and assist the family by helping in household chores. As a result, it eases some of the domestic chore burdens in the home". (Interviewee 8, Male, Muslim)

\section{The impact of identity on families' meal social interaction behaviour}

The positioning or ranking of an individual within the family is a core identity issue highlighted by all participants irrespective of their gender or religious background, as influencing their families' meal social interaction behaviour. It was widely acknowledged that, understanding the position or ranks of individual can foster orderliness and respect, which determines the order of meal sharing. However, using positioning or ranking in defining identity at mealtimes was unanimous accepted as discriminatory, and significantly causes alienation of nonfamily members at the dinner table.

"It depends on the family at the dinner table. If it is just the immediate family like mom, dad, sisters and brothers, but if the dinner table includes extended family, identity definition like I said brings discrimination. At times, it is important to do, as that is how respect comes in, as you know this is your dad or mom, and you cannot talk to them anyhow and you need to give them the respect they deserve. Mom and Dad usually take the lead at the dinner table in anything that you are doing and the children will follow". (Interviewee 39, Female, Muslim)

"Whilst you are at the dinner table and they have identified you as the figure head, even though they may give premium to the eldest, in terms of meal, they feel you should be the first to taste/try the meal. They give you that priority. Once they have finished cooking, if it is chicken, for example, they will say let give daddy the priority. Even the eldest will tend to identify you as the one they should be given respect to and that kind of thing". (Interviewee 22, Male, Muslim)

\section{Discussion}

The present study provides the first exploratory perspective of the influence of reference groups on families' meal social interaction behaviour in Sierra Leone. In the context of age, the researcher found out that all the participants (Muslim 
and Christian females, and Muslim and Christian males) emphasized respect, hierarchy and authority as key influencers of families' meal social interaction behaviour. However, despite these similarities, Muslim females and their male counterparts, and Christian females reiterated the symbolic effect of wisdom on their families' meal behaviour. In addition, Muslim females and males were more emphatic about experience as a major age factor influencing their families' meal behaviour, a factor less emphasized by the Christian females and their male counterparts. It is evident that, within the same religion and gender groups, differences exists in opinions about the impact of age on families' meal behaviour, including: learning; advice; decision-making; peace/harmony; maturity; love; protection; understanding; contentment; unity/stability; tolerance; contribution; success; blessing; values; affection; and participation. This suggests that despite age being central to the meal behaviour of families; they views were not generic across families. The findings suggests that, older-generation family members are seen as a symbol of respect, who take food before the younger ones, and on formal occasions when guests are present children may be excluded from the dining table until the adults are finished, or are seated at a table separate from the adults. However, the findings of this study highlighted authority, wisdom, experience and hierarchy as specific age influencing factors in families' meal social interaction behaviour.

Gender was a non-influencing factor in families' meal social interaction behaviour. This is due to the fact that, gender is a discerning and discriminatory instrument in families at mealtimes. Differences in opinion were evident even among families of the same religious and/or gender groups, including: role definition; male supremacy; breadwinner; learning; boundaries; authority; respect; and food quality. This implies participants have a neutral opinion of the influence of gender on their families' meal behaviour. This further suggests that as families migrate and acculturate, there are greater tendency for them to ignore the traditional values of their upbringing and embrace new ones, and this trend is likely to continue in the future as more people become oriented about its negative effect. This perspective of the transitional view of gender has been echoed by past theorists and highlights the differing levels of susceptibility to reference group influences. This implies that families are gradually showing disdain for gender discrimination at mealtimes, and that there are greater tendencies that this cultural stigma will fade away in the long-term [21-23].

Associates from the perspectives of all participants, play a critical role in fostering closer relationship with the family members at mealtimes. However, Muslim females, Christian females and males emphasized idea sharing as a fundamental influencing factor of associates. In addition, Muslim and Christian females proclaimed that associates are critical in regulating the behaviour of their children, which may be due to gender roles relating to socialization and education. The importance of associate was consistent across religious and ethnic groups, but differences in opinion exists, including: respect; troubleshooting; affection; unity/stability; breed conflict; advice; burden on the families' resources; civility; thieving; love; prayers; expectations; source of blessing; family image; family history; identity; politeness; participation; guidance; values; and forum for socialization.
This implies that, associates foster closer bond and idea sharing with the family at mealtimes. They are also shapers of children's behaviour at mealtimes. Muslim and Christian females' appreciation of building closer ties, sharing ideas and shaping the behaviours of the children, could be attributed to the fact that, they are more engaged and involved in managing the welfare of the homes.

Decision-making is crucial to the planning of families' meal purchases and management of the homes, and act as a voice of authority or control, especially in reigning in the behaviour of the children at mealtimes. It also plays a fundamental role in unifying the family and symbolic in reinforcing hierarchy/ authority at mealtimes. In addition, Muslim females emphasized that decision-making contributes to their families' development and act as an instrument of respect within the family. However, differences do exist even within the same religious and/or gender groups, including: consultation; progress/success; prioritizing; peace; age; care; organizing; task distribution; responsibility; harmony; direction; safeguards; discipline; participation; learning; equality; achievement; choices; obedience; harmony; management; coordination; family image; understanding; waste reduction; consent; judgment; rationality; contribution; collaboration; learning; stability/unity; and democracy. This suggests that decision-making is fundamental to planning, management and authority or control at mealtimes. Participants view decision-making as significant to families' development and foster respect at the dinner table. Despite the collective nature of decision-making, the final decision rests with the breadwinner(s) of the family-usually the husband/ dad, but wives and eldest son/daughter make decisions in the absence of the former. This study suggests that, children learn to take their bearings from others when it comes to decisionmaking, and that personal decision-making does not existthey are pre-determined by the group or family. However, this study highlighted planning; authority; family unity; and family development as influencers of families' decision-making at mealtimes. Invariably, the inability of the head of the family to make the right decisions may affect the survival of the family.

Background differences emerged as key influencer of extended families involvement in families' meal social interaction behaviour. The differences in background can be attributed to variation in cultural, behavioural and/or upbringing of individuals in the family. However, Muslim females, Christian females and Muslim males reiterated that financial and/or economic burden is a major impact of extended families on their families' meal behaviour. In addition, Muslim females and males emphasised that, participation and facilitation of the performance of domestic chores at mealtimes is a primary benefit of extended families. Differences emerged even within the same religious and/or gender groups, including backbiting; divulge family secret; breeds hatred; stealing; witchcraft; sharing; conflict; advice; jealousy; lineage; knowledge; behavioural challenges; domino effect; obligation; ostracisation; stalling development; malice; income subsidisation; charity; cultural beliefs; bond strengthening; bullying; appreciation/gratification; and gifts. Previous research suggested that, families in collectivist societies, display distinct value attributes completely different from those of extended family members, because of the variation in backgrounds and culture [24]. In this study, the researcher 
identified both the negative and positive effects of extended families on families' meal behaviour. This implies that despite the negativity, for example, witchcraft associated with extended family, there are a number of positive benefits, for example, subsidization of the families' incomes, which facilitate easy access to food and resources and the impending domino effect of helping one member have on others.

Positioning/ranking emerged as a key identity factor that influences families' meal social interaction behaviour. However, Muslim females and males see the practice of identity definition at mealtimes as discriminatory and therefore do not embrace it to define the position/rank of individuals at mealtimes due to its adverse effect on extended families or other social relations living with the family and/or sharing meal with the family. Variation in views emerged among participants of the same religious and gender groups, including: role/responsibility; control; respect; expectations; ethics; unity; boundaries; sense of belonging; priority; age; obedience; orderliness; and learning. This suggests that despite its positive impact, identity definition is discriminatory and can serve to alienate non-family members at mealtimes. Past research efforts have examined the complex construct of identity, as one important factor of individual's position in the family, which needs to be broken down into different variables of which it is comprised. In this study, the researcher found out that, identity construct is predominant in most families at mealtimes. This implies that the use of identity in defining the position occupied by individuals in the family can have crucial bearing on their roles, but can also act as a catalyst of behavioural control within the family.

\section{Implications}

The findings of this study highlight the positive and negative effects of reference groups on families' meal social interaction behaviour. Important cultural, economic and social differences and similarities in mealtime behaviours emerged among the different ethnic and religious groups in the areas of age, gender, associates, decision-making, extended families and identity. The implications for the study is that reference groups such as aunts, uncles, cousins and colleagues/friends irrespective of the religious and/or ethnic divide significantly influence the way families interact socially at the mealtimes. Sometimes, reference groups known as out-groups, can be significant influencers of families' decision-making just as the reference groups, known as an in-group, as people in collectivist culture tend to revered their elders, which may have implications on how their families are run. Consequently, the concept of self reflects social relationships and roles, and evidently, any society tailored towards norms and relationship building tends to be highly susceptible to such reference group influences.

The findings of this study suggests that, reference groups are particularly influenced by factors such as hierarchy/authority; role distinction; wisdom; experience; gender differentiation; education; affection; social benefits; neighbours; and family relations. It is imperative from the findings that, despite the benefits of reference groups, there are inherent challenges, including the threat it poses to the way families relate with each other. Future interventions should build on the findings of this study to find ways to address the cultural, social and economic challenges posed by reference groups in families' meal social interaction behaviour. Efforts should also recognize the underlying issues of spiritual beliefs such as witchcraft besieging the mind-sets of certain families with regards the influence of references and highlight how to address it.

\section{Conclusion}

Participants overwhelmingly suggested that, reference groups affect their families' meal social interaction behaviour. However, despite similarities in views among participants of the impact of reference groups on their families' meal behaviour, there were marked differences. This suggests that, the influence of reference groups on families' meal behaviours is not generic. Consequently, reference groups have been shown to encourage adherence to authority/hierarchy; and provides the opportunity for better family planning, which ensures continuous access to food at all times; and creates a good environment that foster closer ties between family members and external relations when socializing at mealtimes. However, reference groups have also been shown to adversely affect the socio-economic situations of families by limiting their access to adequate and quality food. This study did not support the assertion that gender plays a major role as influencer of families' meal behaviour. The implications of this conclusion are that adherence to authority/ hierarchy can help in identifying and streamlining behavioural appropriateness and clearly define the order of command and control, and the level of respect accorded to individuals at the dinner table. This further implies that the socio-economic costs of reference groups can have adverse effect on the sustainability, stability and survival of families as they struggle to make ends meet.

\section{References}

1. Kirkman BL, Lowe KB, Gibson CB. A retrospective on Culture's Consequences: The 35-year journey. Journal of International Business Studies. 2017 Jan 1;48(1):12-29.

2. Tjosvold D. Cross-cultural management: foundations and future. Routledge; 2017 Mar 2.

3. Lawan LA, Zanna R. Evaluation of socio-cultural factors influencing consumer buying behaviour of clothes in Borno State, Nigeria. Int. J. Basic Appl. Sci. 2013 Jan;1(3):519-29.

4. Ayoko OB, Muchiri M. Conflict at multiple levels: transformational leadership and followers' cultural orientation. Handbook of Conflict Management Research. 2014 Jan 1;440.

5. Abraham A, Abdullah I, Gberie L, M'bayo T, Cole G, Blyden N, Cole F, Bangura Y. The Paradoxes of History and Memory in Post-Colonial Sierra Leone. Lexington Books; 2013 Oct 10.

6. Bolten CE. Social Networks, Resources, and International NGOs in Postwar Sierra Leone. African Conflict \& Peacebuilding Review. 2014;4(1):33-59.

7. Kagitcibasi C. Family, self, and human development across cultures: Theory and applications. Routledge; 2017 Jul 20.

8. Simons H. Case study research: In-depth understanding in 
context. The Oxford handbook of qualitative research. 2014 Apr 7:455-70.

9. Al-Krenawi A. Context and Change: The Structure of Arab Society. InPsychosocial Impact of Polygamy in the Middle East 2014. Springer, New York, NY:25-50.

10. Chinunda ED. Grappling with change in Africa: The dream of prosperity using African wisdom. $4^{\text {th }}$ Edition, Author House, Bloomington 2014.

11. Manyama W. Dynamics in family patterns in Tanzania: The case of Kijitonyama ward, Kinondoni District, Dar Es Salaam Region, Tanzania. Journal of Sociology 2017;5(1), 68-79.

12. Sanchez-Sosa JJ, Poldrugo F. Family and cultural influences on alcohol and young people. Learning about drinking. 2001:57-83.

13. Amato P. Approaches to Measuring Families. InEmerging Methods in Family Research 2014 (pp. 179-193). Springer, Cham.

14. Epstein JL. School, family, and community partnerships: Preparing educators and improving schools. Routledge; 2018 Apr 17.

15. Bonvalet C, Clement C, Ogg J. Renewing the family: A history of the baby boomers. Springer; 2014 Oct 10.

16. Telzer EH, Tsai KM, Gonzales N, Fuligni AJ. Mexican American adolescents' family obligation values and behaviors: Links to internalizing symptoms across time and context. Developmental psychology. 2015 Jan;51(1):75.
17. Waugh E, Szafran O, Triscott JA, Parent R. Cultural Competency Skills for Health Professionals: A Workbook for Caring Across Cultures. Brush Education; 2015 Jan 12.

18. Attah R, Barca V, Kardan A, MacAuslan I, Merttens F, Pellerano L. Can social protection affect psychosocial wellbeing and why does this matter? Lessons from cash transfers in sub-Saharan Africa. The Journal of Development Studies. 2016 Aug 2;52(8):1115-31.

19. Daya S. Ordinary ethics and craft consumption: A Southern perspective. Geoforum. 2016 Aug 1;74:128-35.

20. Horner S, Swarbrooke J. Consumer behaviour in tourism. Routledge; 2016 Apr 28.

21. Bhanot S, Srinivasan R, Srivastava RK. Influence of ethnicity on uniqueness \& snob value in purchase behaviour of luxury brands. Journal of Research in Marketing. 2014 Jun 28;2(3):172-86.

22. Jamal A, Shukor SA. Antecedents and outcomes of interpersonal influences and the role of acculturation: The case of young British-Muslims. Journal of Business Research. 2014 Mar 1;67(3):237-45.

23. Segev S, Ruvio A, Shoham A, Velan D. Acculturation and consumer loyalty among immigrants: a crossnational study. European Journal of Marketing. 2014 Sep 2;48(9/10):1579-99.

24. Cloward RA, Ohlin LE. Delinquency and opportunity: A study of delinquent gangs. Routledge; 2013 Aug 21. 